\title{
AVALIAÇÃO DE CADEIAS DE SUPRIMENTOS SUSTENTÁVEIS: O CASO DOS OPERADORES LOGÍSTICOS BRASILEIROS
}

\author{
SUSTAINABLE SUPPLY CHAIN EVALUATION: THE CASE OF BRAZILIAN \\ LOGISTICS OPERATORS
}

Recebido: 22/02/2017 - Aprovado: 14/04/2017 - Publicado: 27/06/2017 Processo de Avaliação: Double Blind Review

\author{
Maciel Manoel de Queiroz ${ }^{1}$ \\ Doutorando em Engenharia Naval e Oceânica \\ Universidade de São Paulo- USP \\ Mestre em Engenharia Naval e Oceânica \\ maciel.queiroz@usp.br \\ Fátima Guarda Sardeiro \\ Mestrado em Educação Matemática \\ Centro Universitário Senac - Santo Amaro \\ fatima.gsardeiro@sp.senac.br \\ Maria Carolina C. Cunha Carneiro \\ Doutora em Educação \\ Centro Universitário Senac - Santo Amaro \\ maria.ccarneiro@sp.senac.br
}

Escola Politécnica da Universidade de São Paulo - Departamento de Engenharia Naval e Oceânica

RESUMO: O trabalho destaca contribuições para que as organizações possam tomar decisões na escolha de parceiros de negócios visualizando um conjunto de variáveis sustentáveis e o possível comportamento que a cadeia de suprimentos poderá estar condicionada. Para isso, foi realizada uma pesquisa exploratória, contemplando uma pesquisa bibliográfica, bem como um questionário encaminhado a onze operadores logísticos brasileiros. Os resultados apontam que as empresas pesquisadas possuem certificações que contemplam as questões ambientais, porém, percebe-se que são ações que priorizam mais a questão de regulamentação, porque, além de serem certificações que,

\footnotetext{
${ }^{1}$ Autor para correspondência: Universidade São Paulo - Avenida Professor Melo Morais, 2231- Cidade Universitária, São Paulo SP Brasil - CEP 05508-900.
} 
se não adotadas resultam em penalidade, não há um gerenciamento integrado de fluxos destas ações com os demais envolvidos no processo pela falta de inspeção periódica.

Palavras-chave: Cadeias de Suprimentos Sustentáveis; Critérios de avaliação; Variáveis sustentáveis.

ABSTRACT: This paper highlights contributions that organizations can make decisions when choosing business partners viewing a set of sustainable variables and the possible behavior that the supply chain can be conditioned. For this, an exploratory research was conducted, contemplating a literature review and a questionnaire sent to eleven Brazilian logistics operators. The results show that the surveyed companies have certifications that address environmental issues, however, one realizes that they are actions that most prioritize the issue of regulation, because in addition to being certified which, if not taken result in a penalty, there is no management integrated flows of these actions with others involved in the process by the gap of periodic inspection.

Keywords: Sustainable Supply Chain; Evaluation criteria; Sustainable variables.

\section{INTRODUÇÃOO}

As cadeias de suprimentos sustentáveis (sustainable supply chain management - SSCM) têm se tornado um dos tópicos de pesquisa mais relevantes na área de supply chain e logística, tanto no aspecto da indústria, quanto no acadêmico (BRANDENBURG et al., 2014; FAHIMNIA; SARKIS; ESHRAGH, 2015; KUEI, et al., 2015; WU et al., 2015; JAYARAM; AVITTATHUR, 2015). As formas de governança (gerenciamento) são um fator fundamental para que determinada cadeia possa atingir significativa performance em diversos indicadores, não somente em tradicionais, como o de custos e qualidade, mas também em relação aos aspectos sociais, ambientais e econômicos. Isto requer uma coordenação dos objetivos de toda a cadeia para a otimização da performance no horizonte de tempo especificado (CARTER; JENNINGS, 2002; BARBIROLI; RAGGI, 2003).

A integração de aspectos sociais, ambientais e econômicos forma o tripé que compõe o conceito de sustentabilidade. Esta concepção é conhecida como o triple bottom line (TBL) de sustentabilidade organizacional (ELKINGTON, 2004) e ganhou relevância nas últimas décadas para a tomada de decisão nas cadeias de suprimentos. 
Estudos sugerem que a seleção de fornecedores pode ser baseada em critérios relacionados a práticas ambientais (HUMPHREYS; WONG; CHAN, 2003) e na gestão de materiais perigosos (HSU; HU, 2009). Outros estudos destacaram as práticas de sustentabilidade social, econômica e ambiental, como os critérios para a seleção de fornecedores (GOVINDAN; KHODAVERDI; JAFARIAN, 2013) e abordagem das cadeias de suprimentos mais sustentáveis (SEURING, 2013).

Adicionalmente, segundo Kannan et al. (2014) a seleção de fornecedores com base em critérios ambientais tem atraído a atenção de muitos pesquisadores. Desta forma, destacam-se alguns estudos como: Jabbour e Jabbour (2009) que verificaram se as empresas brasileiras adotam exigências ambientais de seleção com base nos fornecedores: (1) Custo; (2) Qualidade; (3) Inovação; (4) Entrega; (5) Restrições à utilização de produtos químicos e (6) ISO 14001.

Diante deste contexto, a questão norteadora deste trabalho é: Quais os critérios usados para avaliar cadeias de suprimentos sustentáveis na perspectiva de operadores logísticos que atuam no Brasil? Nesse sentido, o principal objetivo é avaliar quais são as práticas sustentáveis mais adotadas e relevantes nas cadeias de suprimentos sustentáveis na perspectiva de operadores logísticos. Desta forma, os objetivos específicos desta pesquisa são:

i. Analisar e compreender a sustentabilidade das cadeias de suprimentos dos operadores logísticos do Brasil;

ii. Avaliar as ações adotadas e mapear oportunidades de melhorias para o setor.

O restante do artigo está organizado da seguinte forma: na próxima seção é apresentado o referencial teórico deste estudo. Na seção seguinte, a metodologia é destacada, seguida da discussão dos resultados e na sequência, as considerações finais.

\section{1 - REFERENCIAL TEÓRICO}

A literatura brasileira sobre green supply chain management (GSCM) ainda é pequena em relação ao exterior, porém, nos últimos anos, este tema tem chamado a atenção de diversos pesquisadores e tem surgido trabalhos interessantes sobre diversas abordagens das cadeias de suprimentos sustentáveis. Por exemplo, vantagem competitiva em cadeias de suprimentos sustentáveis (BRITO; BERALDI, 2010), análise de sustentabilidade em cadeias de suprimentos (DALÉ; ROLDAN; HANSEN, 2011), green supply chain na perspectiva da indústria canavieira 
(ANDRADE; PAIVA, 2012), inovações socioambientais (CARVALHO; BARBIERI, 2013), e difusão das práticas de green supply chain (ALVES; NASCIMENTO, 2014)

As cadeias de suprimentos sustentáveis, embora seja fundamental compreender tanto os aspectos operacionais, ambientais e econômicos, Silva et al., (2013) reconhece que há pouca literatura publicada no Brasil, principalmente em periódicos. Os autores destacaram que, como no contexto internacional, ocorre uma tendência de crescimento de investigações sobre o tema, que abrange o conceito e como aplicá-lo corretamente no estudo das cadeias de suprimentos, inclusive a brasileira.

Uma cadeia para ser sustentável deve ter um bom desempenho em todos os elementos do Triple Bottom line (PAGELL; WU, 2009). Dessa forma, ações específicas, como decisões gerenciais, devem ser tomadas para que isso ocorra, ou seja, para ser mais sustentável. Nesta perspectiva, destacam que novos valores devem ser incorporados ao longo das cadeias de suprimentos, como integração de novos valores e capacidades, alinhando elementos ambientais e sociais de sustentabilidade.

Uma das estratégias que os operadores logísticos têm utilizado para a criação de uma cultura sustentável que permeie por toda a cadeia de suprimentos, é por meio de certificações. As certificações, fazem parte das decisões estratégicas e gerenciais dos operadores e que devem funcionar de modo que possa difundir e multiplicar as boas práticas, assim como procurar novas formas de desenvolver processos que minimizem o impacto negativo, na esfera ambiental, econômico e social.

De acordo com Silva et al. (2013), dentre os estudos brasileiros relacionados ao tema sustentabilidade, poucos deles contemplam cadeias de suprimentos de maneira crítica. A perspectiva apresentada é, na maioria das vezes, bibliométrica. Verificou-se que há a predominância de estudos de caso, enfocando cadeias de suprimentos do setor primário (64\%), seguidas pelas pesquisas bibliográficas $(36 \%)$. Como não foram localizados estudos brasileiros que usem outros tipos de pesquisa (secundária, survey, Grupo Focal, experimento), estes podem se apresentar como possibilidades de avanço nesta área, trazendo novos elementos. Além disso, é importante que se estimulem pesquisas envolvendo cadeias de suprimentos do setor secundário, em função da demanda de recursos energéticos, materiais e do potencial de impacto nas três dimensões do triple bottom line.

Adicionalmente, Rauer e Kaufmann (2015) afirmam que pesquisadores identificaram diversas barreiras internas e externas para a implementação bem-sucedida de GSCM das empresas (por exemplo, custos associados à GSCM, fornecedores resistentes, distintas regulamentações entre países, etc.). Contudo, estes pesquisadores afirmam haver lacunas na literatura da área em como as empresas lidam com estes obstáculos, ou até mesmo, a investigação do papel das capacidades 
dinâmicas em atenuá-los. Assim, é importante abordar a questão crítica de como as empresas podem tornar suas cadeias de suprimentos verdadeiramente sustentáveis frente aos desafios enfrentados quando tentam implementar práticas sustentáveis, como GSCM.

Ainda segundo Rauer e Kaufman (2015) e em outras palavras, o foco de sua investigação residiu em como as empresas podem efetivamente reduzir ou superar tais barreiras à GSCM e aumentar a sustentabilidade ambiental de suas cadeias de suprimento. Identificaram em seu estudo, duas grandes categorias de barreiras externas relativas à implementação bem-sucedida de GSCM em empresas: as barreiras relacionadas com a estrutura da cadeia de suprimento, e relacionadas com as normas ambientais. Observaram ainda, três categorias de mitigação relativas às capacidades: capacidades de detecção, capacidade de alinhamento e as capacidades de resiliência. Nesse sentido, as capacidades dinâmicas podem reconfigurar processos operacionais (HELFAT et al., 2007), este estudo assume que as empresas aplicam distintas capacidades dinâmicas como parte de seus processos e rotinas organizacionais utilizados para reduzir as barreiras à implementação GSCM.

Em relação as capacidades de alinhamento, a presente pesquisa explora esta variável, em especial com uma questão do questionário aplicado, em que é verificado se a empresa é cobrada por algum programa de gestão ambiental ligado a algum fornecedor ou prestador de serviço. Esta questão é muito importante, pois, como as cadeias de suprimentos são integradas, é crucial que haja uma cobrança por outro elo da cadeia, de modo que o operador possa receber um feedback e promover ações de melhoria.

Grant (2013) afirma que, no sentido de tornar a cadeia de suprimentos mais sustentável, o World Economic Forum sugere que os esforços colaborativos encontram-se em três grupos sendo: i) os prestadores de serviços logísticos e transporte; ii) expedidores e compradores como recebedores de tais serviços; iii) e organizações (governamentais e não governamentais) que instituem políticas. Diante do apresentado por Silva et al. (2013), há uma escassez de pesquisas sobre cadeias de suprimentos sustentáveis de maneira crítica, sendo que a presente pesquisa situa-se no setor terciário e trata da prestação de serviços logísticos, tanto de execução, quanto na gestão de atividades de abastecimento, armazenagem e transportes, denominados PSL (Operadores Logísticos ou Prestadores de Serviços Logísticos).

A demanda por este tipo de serviços, de acordo com Novaes (2004) foi estimulada por mudanças globais impulsionadas por alguns fatores, tais como: o término da primeira e segunda guerras mundiais e a necessidade de reconstrução de boa parte do mundo; a criação de organismo internacional que regulamentasse o comércio entre os países e uma predisposição para eliminação de barreiras que impedissem tal prática; e o desenvolvimento da tecnologia da informação. Para este 
pesquisador, todos estes fatores levaram ao crescimento do comércio entre as empresas e a expansão desta além das fronteiras nacionais, tornando mais complexa sua logística. Aliado a este fator, a necessidade por redução de custos, porém mantendo um nível de serviços excelente, estimularam a busca por prestadores de serviços especializados.

Novaes (2004) ainda destaca que, no Brasil, o PSL tem origem, em grande parte nos transportadores (Águia Branca, Expresso Joaçaba, Ouro e Prata, Transportadora Cometa, entre outros) e também na formação de alianças entre os operadores logísticos estrangeiros (Ryder, Danzas, TNT Logistics) com empresas brasileiras prestadoras de serviços, dando origem a grandes Operadores Logísticos. Estes são capazes de oferecer serviços de transportes, armazenagem, manipulação de produtos, operações industriais e comerciais, serviços de cunho informacional e consultoria em engenharia e administração logística. Dado crescimento, complexidade e importância deste setor, veio a necessidade da criação de um organismo que representasse o segmento. Com este objetivo foi criada, em julho de 2012, a Associação Brasileira de Operadores Logísticos (ABOL) que tem a missão de representar os operadores logísticos no Brasil para que gerem valor de forma sustentável.

\section{METODOLOGIA}

A presente pesquisa emprega o método estudo de caso, que é definido por Yin (2006) como uma investigação empírica que investiga um fenômeno contemporâneo dentro de seu contexto da vida real, ou seja, há uma grande contribuição para a realidade atual proporcionada pelos dados que são do cotidiano em que se está inserido, bem como quando os limites entre o fenômeno e o contexto não estão claramente definidos. O estudo de caso ajuda a desenhar um paralelo entre o que está sendo estudado e o contexto.

Na visão de Yin (2006) a investigação de estudo de caso procura enfrentar uma situação única e que terá mais variáveis de interesse do que dados, porque quando estes são analisados, identificamse mais variáveis. Além disto, é beneficiado pelo desenvolvimento de outras proposições teóricas para que haja a coleta e análise dos dados. E, também, é muito utilizado em conjunto com as outras bibliografias, para traçar algum paralelo.

De acordo com Gil (1999) o estudo de caso é caracterizado pelo estudo exaustivo de um, ou poucos objetos, para permitir um conhecimento detalhado. O estudo de caso, pode ser usado em diversos tipos de pesquisas, como descritivas, explicativas e exploratórias. No presente artigo, foram 
selecionados onze dos 127 maiores operadores logísticos brasileiros de 2015, publicado pela revista Tecnologística ${ }^{2}$.

Na visão de Yin (2006) é necessário que haja uma percepção para fazer boas perguntas, porque a pesquisa é baseada principalmente em perguntas, e não necessariamente em respostas. Pode-se classificar esta pesquisa, quanto aos objetivos, de acordo com Gil (2007) como exploratória, já que tem como objetivo proporcionar maior familiaridade com o problema, com vistas a construir hipóteses. Desta forma, foram feitos: um levantamento bibliográfico; questionário com profissionais que tiveram experiências práticas com o problema pesquisado.

Foi enviado um questionário (vide framework a seguir) para onze dos maiores operadores logísticos do País, que se disponibilizaram a participar da pesquisa. 
Figura 1 - Framework Green Supply Chain Model.



Elaborado pelos autores.

\section{DISCUSSÃO DOS RESULTADOS}

Os resultados apontam que mais de $50 \%$ das empresas pesquisadas utilizam certificações emitidas por organismos independentes, como a Fundação Carlos Alberto Vanzolini e Bureau Veritas Quality Internacional (BVQI). Entre as certificações apresentadas estão a ISO 14001 que é atribuída a empresas que querem aprimorar um sistema de gerenciamento ambiental (SGA).

As demais certificações apontadas referem-se a transporte e distribuição de produtos químicos (SASSMAQ- Sistema de Avaliação de Segurança, Saúde, Meio Ambiente e Qualidade) e certificação 
para agir em situações de emergência em acidentes com produtos perigosos (SOS COTEC). Tal fato é confirmado pelos estudos de (HUMPHREYS; WONG; CHAN, 2003) que sugerem que a seleção de fornecedores pode ser baseada em critérios relacionados a práticas ambientais ou, também a gestão de materiais perigosos (HSU; HU, 2009).

Os resultados encontrados sugerem que as empresas buscam uma certificação para atender os requisitos legais para atuar na área de produtos perigosos. Outros estudos destacaram as práticas de sustentabilidade social, econômica e ambiental como os critérios para a seleção de fornecedores (GOVINDAN; KHODAVERDI; JAFARIAN, 2013) no contexto das cadeias de abastecimento mais sustentáveis (SEURING, 2013).

Embora metade das empresas pesquisadas considerem variáveis ambientais (como por exemplo, a ISO 14001), além dos critérios tradicionais (como custo, qualidade, etc.), não há uma inspeção periódica para verificar o desempenho ambiental do fornecedor e assegurar a conformidade com as normas de SGA por $50 \%$ das empresas. Foi apontada uma predisposição para desenvolver produtos e serviços com os clientes para melhorar o impacto ambiental deles $(62,5 \%)$, porém, só se aplicam a $25 \%$ ou menos dos casos apresentados.

Uma constatação importante é que 75\% das empresas incorporam formas de evitar ou reduzir o uso de produtos tóxicos ou perigosos. Dentre as maneiras apresentadas, pode-se citar a utilização de combustível S-10, com baixo teor de enxofre. É importante destacar que tal prática visa atender a legislação específica do PROCONVE (Programa Nacional de Controle da Poluição por Veículos automotores), de acordo com a resolução ANP número 50 de 23/12/2013. A análise de insumos também foi apresentada como uma medida de se evitar ou reduzir o uso de produtos tóxicos ou perigosos. Contudo, tal prática pode estar mais vinculada à garantia de qualidade, uma vez que se trata apenas de análise, e não de tratamento e medidas cabíveis.

De acordo com Grant (2013) o PSL deve buscar alternativas em três áreas, sendo: i) tecnologias; ii) combustíveis e iii) processos novos, no sentido garantir eficiência aos processos, bem como otimizar as infraestruturas utilizadas para o desenvolvimento das operações logísticas. Pode-se ver na pesquisa apresentada que 50\% dos PSLs pesquisados utilizam critérios ambientais tal como uma certificação ao selecionar fornecedores. A mesma situação se configura na inspeção das instalações de fornecedores para verificar o desempenho ambiental desse, onde $50 \%$ fazem esta inspeção. A falta de critérios e inspeções não contribuem com a integração entre os participantes da cadeia de suprimentos, fazendo com que se percam oportunidades de maximizar o aspecto sustentável. 


\section{CONSIDERAÇÕES FINAIS}

O presente artigo abordou um estudo sobre a avaliação das cadeias de suprimentos sustentáveis dos operadores logísticos brasileiros. Procurou-se entender e mapear as práticas sustentáveis deste setor, em especial aos aspectos ligados as ações e estratégias desenvolvidas para minimizar o impacto ambiental.

As importantes evidências são: i) aumento da literatura brasileira sobre cadeias de suprimentos sustentáveis; ii) Criação de um framework para avaliar a sustentabilidade de operadores logísticos; e iii) mapeamento dos principais gaps sobre sustentabilidade em cadeias de suprimentos.

Dentre as principais constatações são que apenas 50\% dos operadores logísticos utilizam certificações emitidas por órgãos independentes, mas já adotam práticas sustentáveis para selecionar fornecedores. Um dos gaps para selecionar fornecedores sustentáveis, é que não há uma inspeção periódica ou indicadores para verificar o desempenho sustentável.

Esta pesquisa possibilita uma extensão com futuros trabalhos como: i) desenvolvimento de indicadores de desempenho sustentáveis para acompanhar a performance dos parceiros de negócios; e ii) verificar se existe relação no aumento da performance sustentável entre os operadores que possuem certificações ambientais.

\section{REFERÊNCIAS}

ABOL- Associação Brasileira de Operadores Logísticos. Site da associação que mostra o mercado de operadores logísticos, boas práticas, marco regulatório e outros dados do setor. Disponível em: < http://abolbrasil.org.br/abol/institucional>. Acesso em: 4 novembro 2015.

ALVES, A. P. F.; NASCIMENTO, L. F. M. Green Supply Chain: protagonista ou coadjuvante no Brasil? RAE-Revista de Administração de Empresas, v. 54, n. 5, p. 510-520, 2014.

ANDRADE, M. C. F.; PAIVA, E. L. Green supply chain management na agroindústria canavieira: o caso Jalles Machado. BASE - Revista de Administração e Contabilidade da Unisinos, v. 9, n. 1, p. 2-12, 2012. 
BARBIROLI, G.; RAGGI, A. A method for evaluating the overall technical and economic performance of environmental innovations in production cycles. Journal of Cleaner Production, v. 11, n. 4 , p. 365-374, 2003.

BRANDENBURG, M.; GOVINDAN, K.; SARKIS, J.; SEURING, S. Quantitative model for sustainable supply chain management: Developments and directions. European Journal of Operational Research, v. 233, n. 2, p. 299-312, 2014.

BRITO, R. P.; BERARDI, P. C. Vantagem competitiva na gestão sustentável da cadeia de suprimentos: um metaestudo. RAE-Revista de Administração de Empresas, v. 50, n. 2, p. 155 $169,2010$.

CARTER, C. R.; JENNINGS, M. M. Logistics social responsibility: An integrative framework. Journal of Business Logistics, v. 23, n. 1, p. 145-178, 2002.

CARVALHO, A. P.; BARBIERI, J. C. Inovações socioambientais em cadeias de suprimento: um estudo de caso sobre o papel da empresa focal. RAI - Revista de Administração e Inovação, v. 10, n. 1, p. 232-256, 2013.

DALÉ, L. B. C.; ROLDAN, L. B.; HANSEN, P. B. Analysis of sustainability incorporation by industrial supply chain in Rio Grande do Sul state (Brazil). JOSCM - Journal of Operations and Supply Chain Management, v. 4, n.1, p. 25-36, 2011.

ELKINGTON, J. Enter the triple bottom line. In A. Henriques \& J. Richardson (Orgs.). The triple bottom line: Does it all add up? London: Earthscan, 2004. p. 1-16.

FAHIMNIA, B.; SARKIS, J.; ESHRAGH, A. A tradeoff model for green supply chain planning: a leanness-versus-greenness analysis. Omega, v. 54, p. 173-190, 2015.

GIL, A. C. Métodos e técnicas de pesquisa social. 5. ed. São Paulo: Atlas, 1999.

GIL, A. C. Como elaborar projetos de pesquisa. 4. ed. São Paulo: Atlas, 2007. 
REMIPE

GOVINDAN, K.; KHODAVERDI, R.; JAFARIAN, A. A fuzzy multi criteria approach for measuring sustainability performance of a supplier based on triple bottom line approach. Journal of Cleaner Production, v. 47, p. 345-354, 2013.

GRANT, D. B. Gestão de logística e cadeia de suprimentos. São Paulo: Saraiva, 2013.

HELFAT, C.; FINKELSTEIN, S.; MITCHELl, W.; PETERAF, M.; SINGH, H.; TEECE, D.; WINTER, S. Dynamic capabilities: Understanding strategic change in organizations. Malden, MA: Blackwell, 2007.

HSU, C. W.; HU, A. H. Applying Hazardous Substance Management to Supplier Selection Using Analytic Network Process. Journal of Cleaner Production, v. 17, p. 255-264, 2009.

HUMPHREYS, P. K.; WONG, Y. K.; CHAN, F. T. S. Integrating environmental criteria into the supplier selection process. Journal of Materials Processing Technology, v. 138, n. 1-3, p. 349-356, 2003.

JABBOUR, A. B. L. S.; JABBOUR, C. J. C. Are supplier selection criteria going green? Case studies of companies in Brazil. Industrial Management \& Data Systems, v. 109, n. 4, p. 477-495, 2009. JAYARAM, J.; AVITTATHUR, B. Green supply chains: A perspective from an emerging economy. International Journal of Production Economics, v. 164, p. 234-244, 2015.

KANNAN, D. Selecting green suppliers based on GSCM practices: Using fuzzy TOPSIS applied to a Brazilian electronics company. European Journal of Operational Research, v. 233, n. 2, p. $432-$ $447,2014$.

KUEI, C-H.; MADU, C. N.; CHOW, W. S.; CHEN, Y. Determinants and associated performance improvement of green supply chain management in China. Journal of Cleaner Production, v. 95, p. 163-173, 2015.

NOVAES, A. G. Logística e Gerenciamento da cadeia de distribuição. 2. ed. Rio de Janeiro: Campus, 2004. 
REMIPE

PAGELL, M.; WU, Z. Building a more complete theory of sustainable supply chain management using case studies of 10 exemplars. Journal of Supply Chain Management, v. 45, n. 2, p. 37-56, 2009.

RAUER, J.; KAUFMANN, L. Mitigating External Barriers to Implementing Green Supply Chain Management: a Grounded Theory Investigation of Green-Tech Companies' Rare Earth Metals Supply Chains. Journal of Supply Chain Management, v. 51, p. 65-88, 2015.

SEURING, S. A review of modeling approaches for sustainable supply chain management. Decision Support System, v. 54, p. 1513-1520, 2013.

SILVA, M. E; NEUTZLING, D. M; ALVES, A. P. F; DIAS, P; SANTOS, C. A. F; NASCIMENTO, L. F. M. Gestão da cadeia de suprimentos sustentável: entendendo o discurso brasileiro. Anais do XXXVII Encontro Nacional da Associação Nacional dos Programas de Pós-Graduação em Administração, Rio de Janeiro, v. 37, p. 1-16, 2013.

WU, K-J.; LIAO, C-J.; TSENG, M-L.; CHIU, A. S. F. Exploring decisive factors in green supply chain practices under uncertainty. International Journal of Production Economics, v. 159, p. 147$157,2015$.

YIN, R. K. Estudo de caso: planejamento e métodos. 3. ed. Porto alegre: Bookman, 2006. 


\section{ANEXO A}

\section{Questionário aplicado:}

1)Há algum programa de auditoria para fiscalizar o cumprimento dos requisitos ambientais legais que demonstra se a sua empresa está tentando atender a regulamentação ambiental para o seu setor internamente?

Sim ( ) Não ( )

Qual?

2) A sua empresa tem uma certificação ISO 14001 ?

Sim ( ) Não ( )

3) Ao selecionar fornecedores há a inclusão de critérios ambientais (ou seja, se considera variáveis ambientais: por exemplo, ISO 14001) além dos critérios tradicionais (custo, qualidade, etc)?

5- Sempre

4- Quase sempre

3-ás vezes

2- Raramente

1- Nunca

4) Há inspeções das instalações de fornecedores como uma maneira de verificar o desempenho ambiental do fornecedor e assegurar a conformidade com as normas de gestão ambiental?

( ) sim ( ) não

4.1) Qual a regularidade?

( ) semestral

( ) anual

( ) bienal

5) A sua empresa procura desenvolver produtos/serviços em conjunto com os clientes para melhorar o impacto ambiental deles?

( ) $\operatorname{Sim}($ ) Não

Até $25 \%$ dos casos ( )

De $26 \%$ até $50 \%$ dos casos ( ) 
Mais de $50 \%$ dos casos ( )

6) A sua empresa compra equipamentos que lhe permitem produzir de maneira mais limpa (sustentável)?

( ) $\operatorname{sim}($ ) não

7) A sua empresa incorpora formas de evitar ou reduzir o uso de produtos tóxicos ou perigosos?

( ) $\operatorname{sim}($ ) não

7.1) Se afirmativo, como?

8) A sua empresa efetua a venda de equipamento usado (após a compra de novos equipamentos) para recuperar seu investimento?

( ) $\operatorname{sim}(\quad)$ não

9) A sua empresa tem mecanismos de avaliação de como os processos de gestão ambiental trouxeram resultados econômicos?

( ) $\operatorname{sim}$ ( ) não ( ) não se aplica

10) Sua empresa é cobrada por algum programa de gestão ambiental ligado a algum fornecedor ou prestador de serviço?

( ) $\operatorname{sim}$ ( ) não ( ) não se aplica 